\title{
Identification of diagnostic and prognostic biomarkers, and candidate targeted agents for hepatitis B virus-associated early stage hepatocellular carcinoma based on RNA-sequencing data
}

\author{
ZHILI ZENG $^{1 *}$, ZEBIAO CAO $^{2 *}$ and YING TANG ${ }^{1,3}$
}

\author{
Departments of ${ }^{1}$ Oncology and ${ }^{2}$ Endocrinology, The First Affiliated Hospital of Guangzhou University of Chinese \\ Medicine, Guangzhou University of Chinese Medicine; ${ }^{3}$ Department of Oncology, Lingnan Medical Research \\ Center of Guangzhou University of Chinese Medicine, Guangzhou, Guangdong 510405, P.R. China
}

Received March 13, 2020; Accepted July 15, 2020

DOI: $10.3892 / \mathrm{ol} .2020 .12094$

\begin{abstract}
Primary liver cancer is a rapidly progressing neoplasm with high morbidity and mortality rates. The present study aimed to identify potential diagnostic and prognostic biomarkers, and candidate targeted agents for hepatitis B virus (HBV)-associated early stage hepatocellular carcinoma (HCC). The gene expression profiles were extracted from the Gene Expression Omnibus database. Differentially expressed genes (DEGs), hub genes and the enrichment of signaling pathways were filtered out via a high-throughput sequencing method. The association between hub genes and the effects of the abnormal expression of hub genes on the rate of genetic variation, overall survival (OS), relapse-free survival (RFS), progression-free survival (PFS) and disease-free survival (DSS) of patients with HCC, as well as pathological stage and grade, were analyzed using different databases. A total of 1,582 DEGs were identified. Gene Ontology analysis revealed that the DEGs were mainly involved in the
\end{abstract}

Correspondence to: Professor Ying Tang, Department of Oncology, Lingnan Medical Research Center of Guangzhou University of Chinese Medicine, 12 Airport Road, Sanyuanli Street, Baiyun, Guangzhou, Guangdong 510405, P.R. China

E-mail: tangying@gzucm.edu.cn

"Contributed equally

Abbreviations: GEO, Gene Expression Omnibus; DEGs, differentially expressed genes; GO, Gene Ontology; KEGG, Kyoto Encyclopedia of Genes and Genomes; AURKB, aurora kinase B; CDK1, cyclin-dependent kinase 1; CDC20, cell division cycle 20; CCNB1/A2, cyclin B1/A2; EHHADH, enoyl-CoA hydratase and 3-hydroxyacyl CoA dehydrogenase; APOA1, apolipoprotein A1; UBB, ubiquitin B; HBV, hepatitis B virus; HCC, hepatocellular carcinoma; RNA-seq, RNA sequencing; OS, overall survival; RFS, relapse-free survival; PFS, progression-free survival; DSS, disease-specific survival

Key words: RNA-seq, HCC, HBV, DEGs, hub genes, targeted agents 'oxidation-reduction process', 'steroid metabolic process', 'metabolic process' and 'fatty acid beta-oxidation'. Enrichment analysis of Kyoto Encyclopedia of Genes and Genomes pathways revealed that the DEGs were mainly associated with 'metabolic pathways', 'PPAR signaling pathway', 'fatty acid degradation' and the 'cell cycle'. A total of 8 hub genes were extracted. Additionally, the abnormal expression levels of hub genes were closely associated with the OS, RFS, PFS and DSS of patients, the pathological stage and the grade. Furthermore, abnormal expression levels of the 8 hub genes were found in $>30 \%$ of all samples. Several small molecular compounds that may reverse the altered DEGs were identified based on Connectivity Map analysis, including phenoxybenzamine, GW-8510, resveratrol, 0175029-0000 and daunorubicin. In conclusion, the dysfunction of fat metabolic pathways, the cell cycle, oxidation-reduction processes and viral carcinogenesis may serve critical roles in the occurrence of HBV-associated early stage HCC. The identified 8 hub genes may act as robust biomarkers for diagnosis and prognosis. Some small molecular compounds may be promising targeted agents against $\mathrm{HBV}$-associated early stage $\mathrm{HCC}$.

\section{Introduction}

Primary liver cancer is a rapidly progressing neoplasm with high morbidity and mortality rates (1). Liver cancer ranks fourth among the most common cancer-related causes of death and sixth in terms of incidence (2). Worldwide, in 2019, hepatocellular carcinoma (HCC) accounted for $\sim 80 \%$ of all primary liver cancer cases (1). There are numerous risk factors for $\mathrm{CC}$, including chronic hepatitis B virus (HBV) infection, hepatitis $\mathrm{C}$ virus (HCV) infection, long-term intake of aflatoxin, alcoholism and non-alcoholic fatty liver disease (3). Chronic HBV and HCV infections are the leading causes of HCC, constituting $\sim 80 \%$ of cases worldwide $(1,4)$, but the mechanism of HCC pathogenesis has not been fully revealed. The current treatment methods for HCC have resulted in big improvements in clinical practice, early stage $\mathrm{HCC}$ can be cured via surgical resection, liver transplantation and radiofrequency ablation (5). However, the prognosis of HCC is poor, with a 5 -year survival rate of $<20 \%$ (2). When the location of a 
tumor is challenging (such as near the liver capsule, diaphragmatic top, important bile duct or gastrointestinal tract) and not suitable for the implementation of the aforementioned methods, there are certain cell cycle-associated drugs, such as doxorubicin, cisplatin and mitomycin $\mathrm{C}$, that can be used to chemoembolize the hepatic artery, in a process known as transarterial chemoembolization (TACE). TACE is considered as a remedy for early and very early stage liver cancer (6-11). However, predictors of early stage HCC remain limited. Ultrasound, as the common means for screening and monitoring early stage HCC, can be affected by various factors, such as the skill of the ultrasound operators, the body habitus and liver nodularity of the patient, which can sometimes make a big difference in the results $(12,13)$. Furthermore, with a cut-off value of $20 \mathrm{ng} / \mathrm{ml}$, the specificity of $\alpha$-fetoprotein (AFP) is $80-90 \%$, but the sensitivity is $40-60 \%$, and normal AFP levels are detected in $\sim 30 \%$ of patients with HCC (14). AFP levels can be influenced by cirrhosis or the level of liver inflammation to a certain extent $(12,15)$. Therefore, numerous patients with HCC are diagnosed at the advanced stage, and surgery to remove all the tumor cells is often difficult, leading to a high recurrence rate and a low 5-year survival rate (16). Therefore, early diagnosis and early treatment are of profound importance for patients with HCC.

Gene variation, deletion and abnormal expression are closely associated with the occurrence, metastasis and recurrence of HCC. With the development of molecular biology and genomics, high-throughput sequencing is expected to provide biomarkers for screening and monitoring liver cancer (1). Compared with sequencing technologies, such as gene chip technology, hybridization- or sequence-based approaches, RNA-sequencing (RNA-seq) has a higher sensitivity, accuracy, breadth and depth using the 'next-generation' sequencing technology, making the results more comprehensive and repeatable (17).

The present study aimed to identify the hub genes and signaling pathways closely associated with HBV-associated early stage HCC using RNA-seq technology. Additionally, small molecular compounds that may reverse the altered differentially expressed genes (DEGs) were identified. These hub genes may be used as potential diagnostic and prognostic markers, and the small molecular compounds may be used as promising candidate agents for HBV-associated early stage HCC.

\section{Materials and methods}

Screening dataset and processing raw data. The study design is presented in the form of a flow chart (Fig. 1). The Gene Expression Omnibus (GEO) database (https://www.ncbi.nlm. nih.gov/geo/) is a public genome database and an online tool for downloading experimental and curated gene expression raw data. The GSE124535 dataset (18) from the GEO database was used in the present study, consisting of $35 \mathrm{HBV}$-associated early stage HCC tissues and 35 matched normal tissues, which were obtained from patients in Zhongshan Hospital, Fudan University (Shanghai, China) and Cancer Hospital \& Institute, Peking University (Beijing, China), with the approval of the Research Ethics Committees of these two hospitals (18). And its Sequence Read Archive (SRA) accession number was SRP174991. The SRA (https://www.ncbi.nlm.nih.gov/sra/) is a primary archive of the National Institutes of Health regarding high-throughput sequencing data and is part of the International Nucleotide Sequence Database Collaboration; it is the largest publicly available repository of high-throughput sequencing data and accepts data from all branches of life sciences, as well as metagenomic and environmental surveys. On June 16, 2020, the total bases and size of the SRA had reached $4.2182 \times 10^{16}$ bases and $1.52091 \times 10^{16}$ bytes, respectively. The SRA stores raw sequencing data and alignment information to enhance reproducibility and facilitate novel discoveries through data analysis. Subsequently, RNA-seq raw data of SRP174991, including 35 early stage HCC tissues and 35 matched normal tissues, were downloaded from the SRA. Next, the original binary SRA data was converted into sequencing data as a fastq file. Firstly, reasonable quality control and filtering were performed on the data. Reads length, reads quality, uncertain base number and other cut-off values were set using Cutadapt software (version 2.4) (http://code.google.com/p/cutadapt/); reads and bases with lower quality were screened out and removed, with wow quality defined as the length of read is $<100$ and the quality of read is <25). Secondly, Hisat2 in the hisat2-build program of the Hisat 2 software and to determine the position of chromosomes, so as to determine which gene it corresponds to. Thirdly, after the reads were aligned to the location of the gene in which they were located, the HTSeq software (19) (version 0.12.4) so that the gene expression levels in the samples could be accurately compared. The identification of DEGs was performed using the 'limma' package (20) (version 3.40.4). The cut-off criteria of $\mid \log _{2}$ fold-change $(\mathrm{FC}) \mid>1$ and $\mathrm{P}<0.01$ were considered to indicate a statistically significant difference.

Function and pathway enrichment analyses of DEGs. To further investigate the identified DEGs, the Database for Annotation, Visualization and Integrated Discovery (v6.8; https://david. ncifcrf.gov/) (21) was used for Gene Ontology (GO) enrichment analysis. GO annotation and functional analyses were performed for DEGs. GO functional analysis included analysis of biological processes (BPs), cellular components (CCs) and molecular functions (MFs). BP refers to pathways and larger processes comprising the activities of multiple gene products, $\mathrm{CC}$ indicates where within the cell the gene products are active and MF indicates molecular activities of gene products (21). The Kyoto Encyclopedia of Genes and Genomes (KEGG) Orthology-Based Annotation System (KOBAS) 3.0 (22) database (http://kobas.cbi.pku.edu.cn/) was used for KEGG enrichment analysis. KOBAS is a web-based tool for gene functional enrichment analysis, including corresponding visual pathway diagrams. $\mathrm{P}<0.05$ was set as the enrichment threshold.

Protein-protein interaction (PPI) network construction. To establish the interactions of these DEGs in HCC, a DEG-associated PPI network was constructed using the Search Tool for the Retrieval of Interacting Genes/Proteins database (https://string-db.org.uk/) (23) with a minimum required interaction score of $>0.9$. Interactions were subsequently visualized and analyzed using Cytoscape v3.7.1 (24) after hiding the disconnected nodes.

Identification of hub genes. CytoHubba (version 0.1) (25), a plug-in of Cytoscape v3.7.1, was used to screen hub genes. 


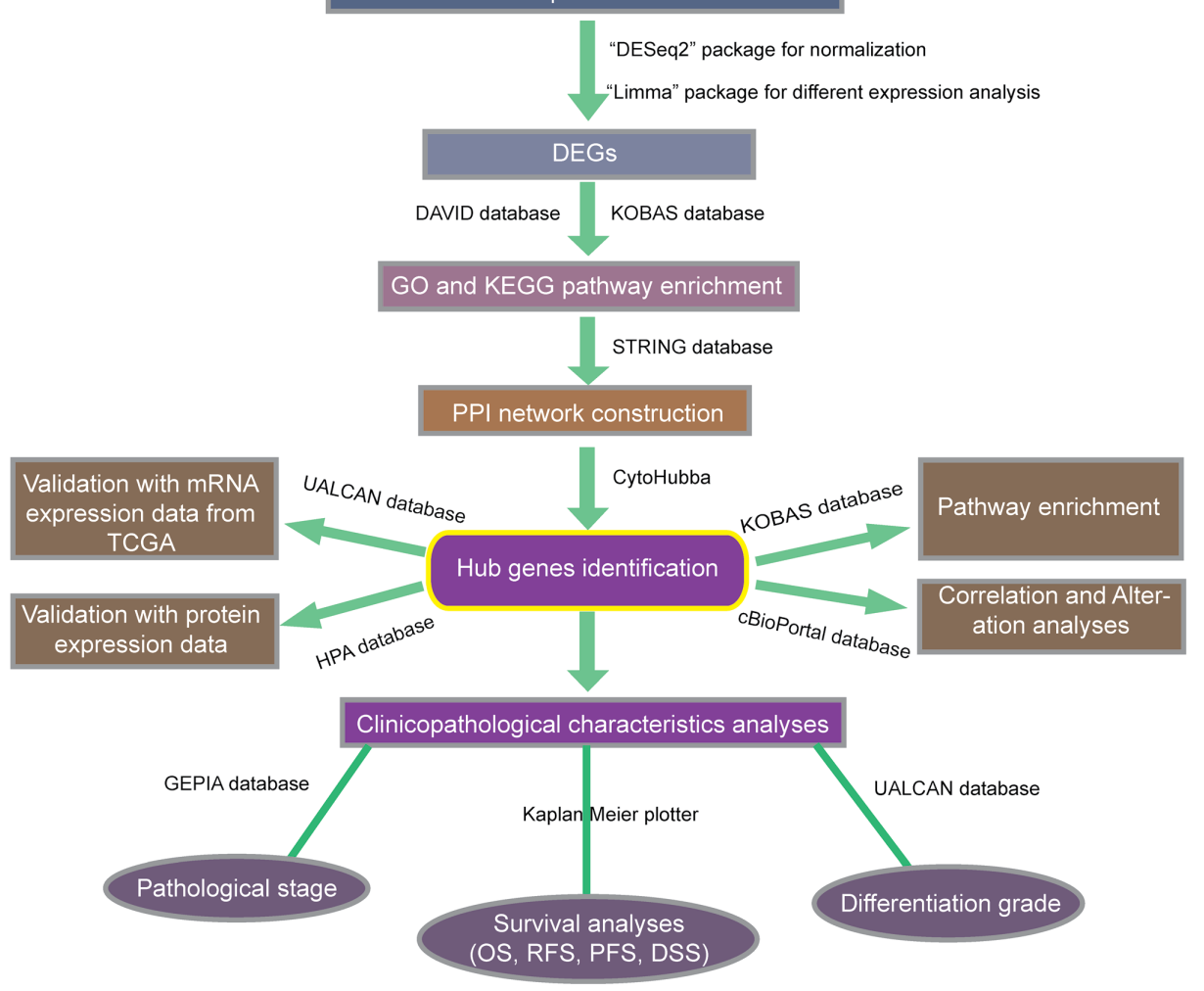

Figure 1. Flow chart of the study. Gene expression profile data were extracted from the GEO database, and DEGs, hub genes and signal pathways were screened by high-throughput sequencing method, and mRNA and protein expression of hub genes were verified in UALCAN database and HPA database respectively. Then, The association between hub genes and the effects of the abnormal expression of hub genes on the rate of genetic variation, OS, RFS, PFS and DSS of patients with HCC, as well as pathological stage and grade, were analyzed using different databases. GEO, Gene Expression Omnibus; DEG, differentially expressed gene; GO, Gene Ontology; KEGG, Kyoto Encyclopedia of Genes and Genomes; PPI, protein-protein interaction; TCGA, The Cancer Genome Atlas; HPA, Human Protein Atlas; OS, overall survival; RFS, relapse-free survival; PFS, progression-free survival; DSS, disease-specific survival; KOBAS, KEGG Orthology-Based Annotation System; STRING, Search Tool for the Retrieval of Interacting Genes/Proteins; SRA, Sequence Read Archive.

Three topological analysis methods of CytoHubba, namely degree, closeness and betweenness, were used to measure each gene in the PPI network. Degree centrality describes the sum of direct edges each node has, and proteins with higher degrees are more likely to be essential proteins (25). Closeness centrality is used to calculate the sum of the distances from one nodes to all other nodes, and betweenness is utilized to calculate the number of shortest paths through a node (26). These three measures are the most commonly used to reveal the relative importance of nodes in the structure of a network (26). The top 50 genes in each analysis method were selected, and the hub genes were filtered from their intersection.

Validating hub genes with $m R N A$ and protein expression data. UALCAN (http://ualcan.path.uab.edu/index.html) (27) is an easy to use, interactive web-portal that can perform in-depth analyses of The Cancer Genome Atlas (TCGA) Program gene expression data (https://www.cancer.gov/about-nci/organization/ccg/research/structural-genomics/tcga). The Human Protein Atlas (HPA) database (http://www.protein atlas. org/) (28) integrates various biotechnologies to cover the database of proteins in almost all human cells, tissues and organs. The mRNA expression levels of the hub genes were verified by the UALCAN online database in 50 normal tissues and 371 HCC tissues from TCGA database. The protein expression levels of the hub genes were verified using the HPA databases. Immunohistochemical (IHC) images were downloaded from the HPA database. The mean integrated optical density (IOD) value of IHC images was measured using Image-Pro Plus software (v6.0; Media Cybernetics, Inc.). The higher the total IOD value was, the greater the expression level was. IHC data were analyzed using an unpaired Student's t-test using GraphPad Prism ${ }^{\circledR}$ v8.0 (GraphPad Software, Inc.). $\mathrm{P}<0.05$ was considered to indicate a statistically significant difference.

Pathway analysis correlation and genetic variation rate of hub genes. The aforementioned KOBAS database was used to conduct pathway enrichment analysis of hub genes. The cBioPortal (http://www.cbioportal.org/) (29) for TCGA provides a web resource for exploring, visualizing and analyzing multidimensional cancer genomics data (29). TCGA contains both sequencing and pathological data of 30 different types of cancer. The HCC study (TCGA, PanCancer Atlas) in the cBioPortal database, containing 372 samples with mRNA data, was used for correlation and genetic change analysis of hub genes according to the online instructions. The data 
type selected was mRNA, and mRNA expression z-scores (RNA Seq V2 RSEM) were selected as the mRNA profile. The correlation between hub genes is represented by Pearson's correlation coefficient (Pearson's correlation coefficient is positive for positive correlation; Pearson correlation coefficient is negative for negative correlation).

Clinical significance of hub genes. The association between hub genes and clinicopathological characteristics was analyzed. The clinicopathological characteristics mainly included the following aspects: Survival analysis, pathological stage and tumor grade. Survival analysis consisted of overall survival (OS), recurrence-free survival (RFS), progression-free survival (PFS) and disease-specific survival (DSS). The Kaplan-Meier plotter (http://www.kmplot.com/analysis/index. php?p=background) (30), and the UALCAN (http://ualcan. path.uab.edu/index.html) databases were used for survival, pathological stage and grade analysis, respectively. The follow up threshold of OS and DSS were set as 60 months. The follow up threshold of RFS and PFS were set as 36 months. Survival analysis was performed using log-rank tests (30). Unpaired t-tests (27) were carried out for stage and grade analysis between groups.

Connectivity map (CMap) analysis. The CMap database (updated on September 12,2017; https://portals.broadinstitute. $\mathrm{org} / \mathrm{cmap} /$ ) (31) is a collection of genome-wide transcriptional expression data from cultured human cells treated with bioactive small molecules and simple pattern-matching algorithms that together enable the discovery of functional connections between drugs, genes and diseases through the transitory feature of common gene expression changes. CMap analysis was utilized to predict underlying small molecule compounds that may reverse the altered expression levels of DEGs in cell lines. Therefore, small molecule compounds with a prominently negative association with the HBV-associated early stage HCC signature were identified and may be potential therapeutic agents for HBV-associated early stage HCC. Mean $<-0.4$ and $\mathrm{P}<0.05$ were set as the threshold values.

\section{Results}

Raw data and identification of DEGs. The GSE124535 dataset was selected, consisting of $35 \mathrm{HBV}$-associated early stage HCC tissues and 35 matched normal tissues, which were obtained from patients in Zhongshan Hospital, Fudan University (Shanghai, China) and Cancer Hospital \& Institute, Peking University (Beijing, China), with the approval of the Research Ethics Committees of these two hospitals. The clinical information of the 35 patients is presented in Table I (18). Among these patients, there were 29 males and 6 females, and their average age is 55, and the age range is 25-80 years. All patients provided early stage (Barcelona Clinic Liver Cancer stages 0 and A) HCC tissues infected with HBV and negative for HCV. Additionally, patients had not undergone prior chemotherapy or radiation. Based on the cut-off criteria, a total of 1,582 DEGs were identified, among which 530 were upregulated and 1,052 were downregulated. Their distribution was presented using a volcano plot (Fig. 2A).
Table I. Clinical information of patients with HCC $(n=35)$.

\begin{tabular}{|c|c|}
\hline Clinical information & Cases, $\mathrm{n}$ \\
\hline \multicolumn{2}{|l|}{ Cirrhosis } \\
\hline Yes & 28 \\
\hline No & 7 \\
\hline \multicolumn{2}{|l|}{ Number of tumors } \\
\hline 1 & 32 \\
\hline 2 & 3 \\
\hline \multicolumn{2}{|c|}{ Diameter of tumor, $\mathrm{cm}^{\mathrm{a}}$} \\
\hline$<3$ & 9 \\
\hline 35 & 25 \\
\hline$>5$ & 1 \\
\hline \multicolumn{2}{|l|}{ Lymphatic metastasis } \\
\hline Yes & 0 \\
\hline No & 35 \\
\hline \multicolumn{2}{|c|}{ Macrovascular invasion } \\
\hline Yes & 0 \\
\hline No & 35 \\
\hline \multicolumn{2}{|c|}{ Microvascular invasion } \\
\hline Yes & 9 \\
\hline No & 26 \\
\hline \multicolumn{2}{|l|}{$\alpha$-fetoprotein, $\mathrm{ng} / \mathrm{ml}$} \\
\hline$<20$ & 18 \\
\hline $20-400$ & 8 \\
\hline$>400$ & 9 \\
\hline \multicolumn{2}{|l|}{ Cancer recurrence } \\
\hline Yes & 3 \\
\hline No & 32 \\
\hline \multicolumn{2}{|l|}{ Died of recurrence } \\
\hline Yes & 0 \\
\hline No & 35 \\
\hline
\end{tabular}

${ }^{\text {a}}$ For multi-nodular cases, the diameter of the largest tumor was listed.

GO and KEGG enrichment analyses. The DEGs in the BP category of GO analysis were mainly enriched in 'oxidation-reduction process', 'xenobiotic metabolic process', 'epoxygenase P450 pathway', 'steroid metabolic process', 'metabolic process' and 'fatty acid beta-oxidation' (Fig. 2B). In the KEGG pathway enrichment analysis, DEGs were significantly enriched in 'metabolic pathways', 'complement and coagulation cascades', 'PPAR signaling pathway', 'fatty acid degradation', the 'cell cycle' and 'chemical carcinogenesis' (Fig. 2C).

PPI network and hub genes. A PPI network of DEGs was established, containing 1,462 nodes, 4,744 edges and an average point degree of 6.49 (data not shown). The analyses of degree, closeness and betweenness in the PPI network were performed using CytoHubba. Subsequently, 8 hub genes were selected from the intersection of the top 50 genes of the three topological analysis methods using the Cytoscape plug-in CytoHubba, among which the upregulated hub genes were 
A

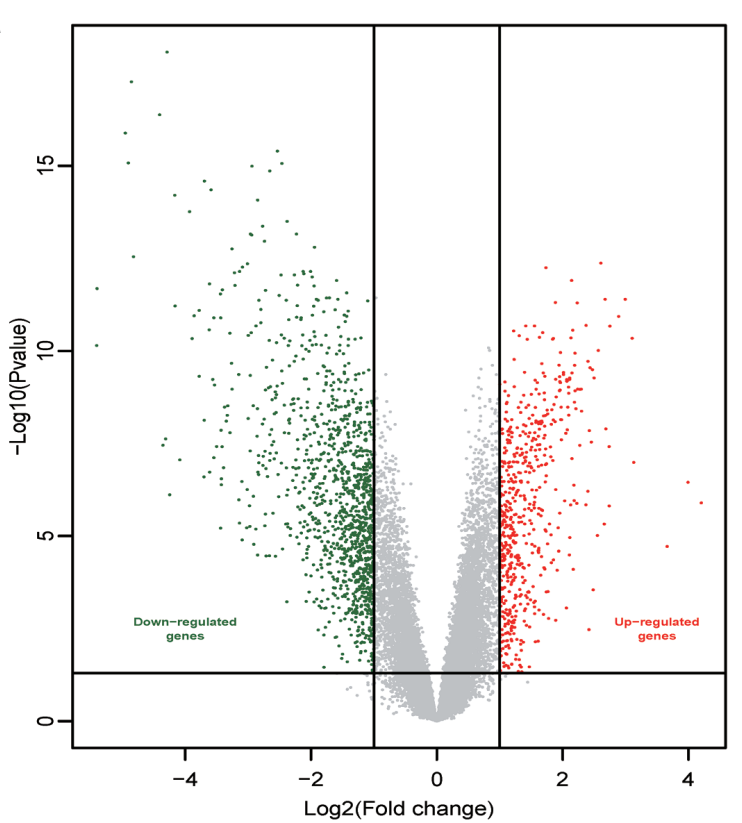

C

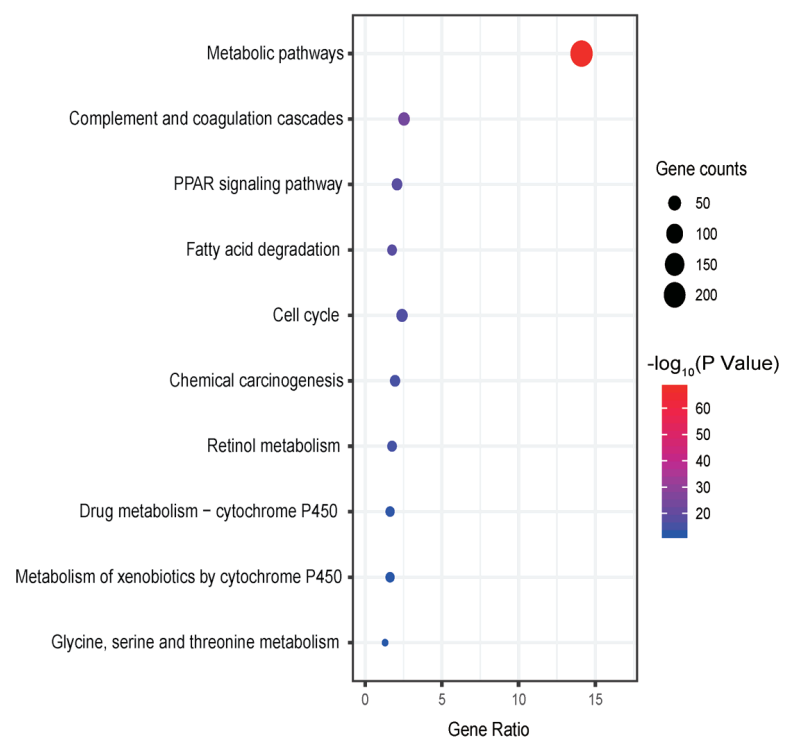

$\mathrm{B}$

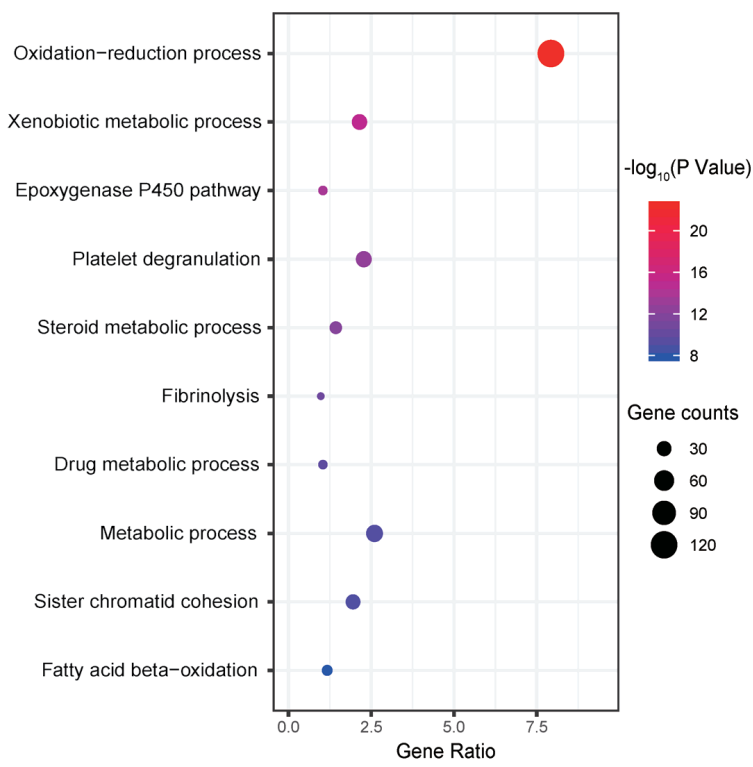

D

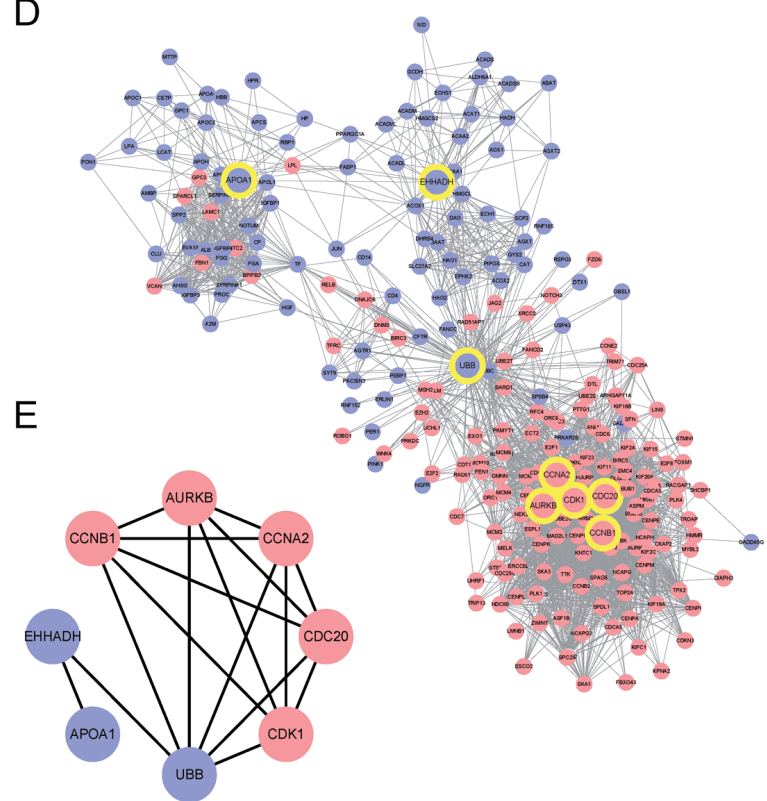

Figure 2. Distribution of DEGs, GO and KEGG enrichment and PPI network. (A) Volcano plot of the distribution of DEGs. (B) Top 10 GO biological processes analysis. (C) Top 10 terms of KEGG enrichment analysis. (D) PPI network of genes closely associated with hub genes. The red nodes represent upregulated genes, the blue nodes represent downregulated genes, the yellow circles are the positions of the 8 hub genes in the PPI network diagram. (E) PPI network of hub genes. AURKB, aurora kinase B; CDK1, cyclin-dependent kinase 1; EHHADH, enoyl-CoA hydratase and 3-hydroxyacyl CoA dehydrogenase; CDC20, cell division cycle 20; CCNA2/B1, cyclin A2/B1; APOA1, apolipoprotein A1; UBB, ubiquitin B; DEGs, differentially expressed genes; GO, Gene Ontology; KEGG, Kyoto Encyclopedia of Genes and Genomes; PPI, protein-protein interaction.

aurora kinase B (AURKB), cyclin-dependent kinase 1 (CDK1), cell division cycle 20 (CDC20), cyclin (CCN)B1 and CCNA2, while the downregulated hub genes were enoyl-CoA hydratase and 3-hydroxyacyl CoA dehydrogenase (EHHADH), apolipoprotein A1 (APOA1) and ubiquitin B (UBB). The PPI network of genes closely associated with the 8 hub genes, with 244 nodes and 2,781 edges, is presented in Fig. 2D. The PPI network between hub genes is shown in Fig. 2E.

Validation of hub genes. The UALCAN online database was used to verify the mRNA expression levels of the 8 hub genes. The protein expression levels of the hub genes were analyzed using IHC samples from the HPA online database. As shown in Fig. 3A-C, the mRNA and protein levels of AURKB, CDK1, CDC20, CCNB1 and CCNA2 were upregulated in tumor tissues compared with in normal tissues, while the mRNA and protein levels of EHHADH, APOA1 and UBB levels were downregulated in tumor tissues (Fig. 3C), indicating that the mRNA and protein expression of hub genes were similar in different databases.

Pathway analysis, correlation and genetic variation rate of hub genes. Hub genes were significantly enriched in signaling pathways such as the 'cell cycle', 'viral carcinogenesis', the 'P53 
A
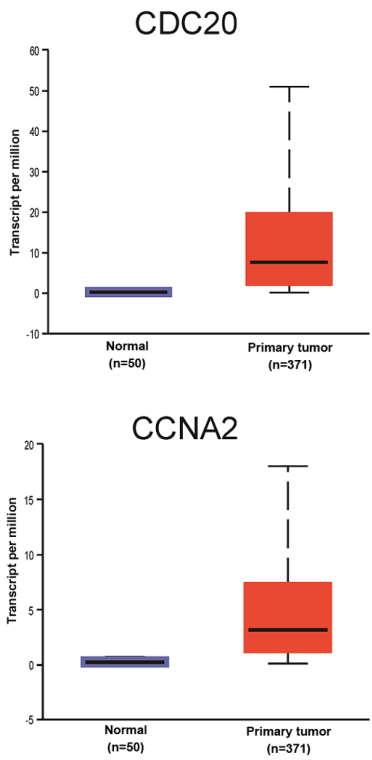

CCNB1

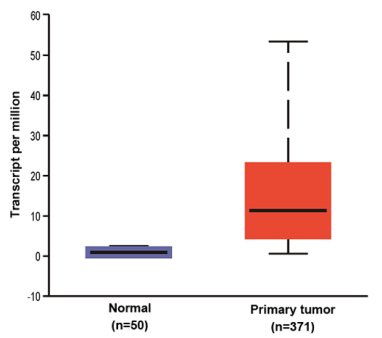

APOA1

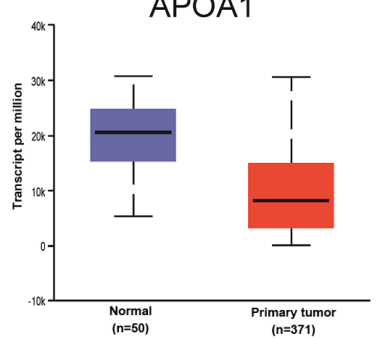

CDK1

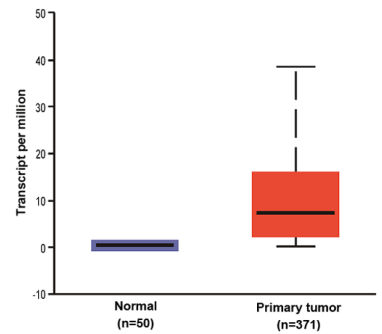

UBB

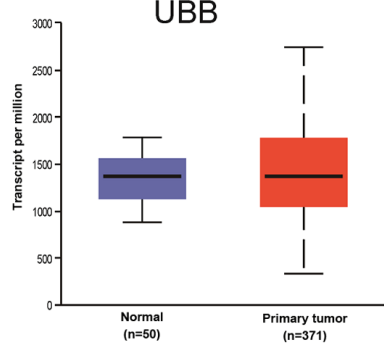

AURKB

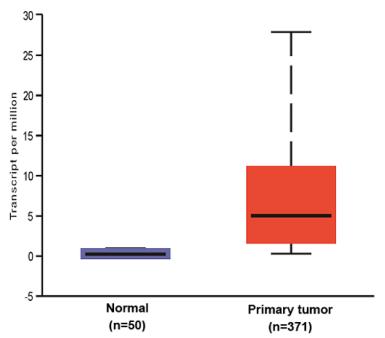

EHHADH
B
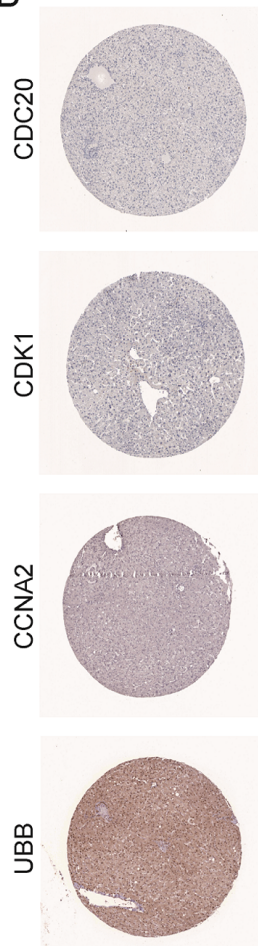

Normal
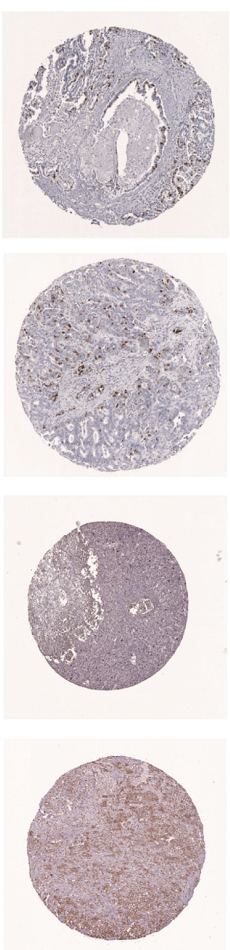

LIHC
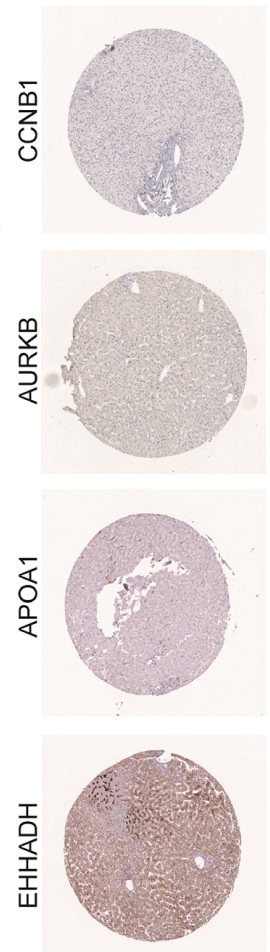

Normal

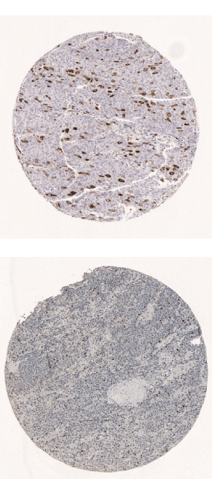

C
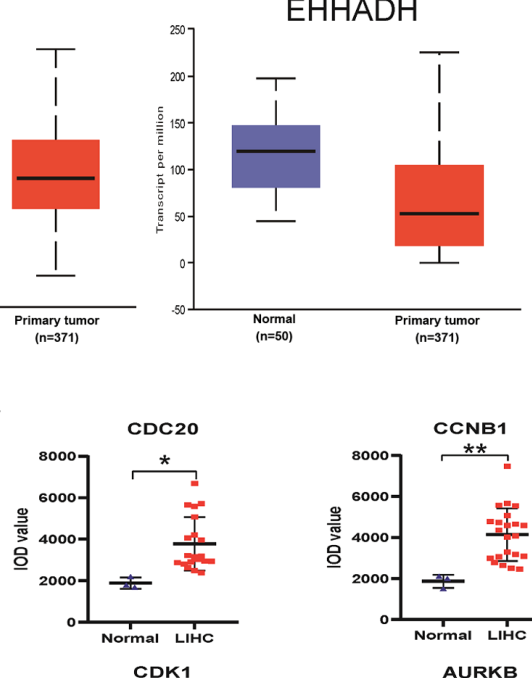

Figure 3. Validation of hub gene expression. (A) Validation of hub gene mRNA expression in The Cancer Genome Atlas database. (B) Validation of protein expression levels of hub genes in the Human Protein Atlas database. AURKB: https:/www.proteinatlas.org/ENSG00000178999-AURKB/tissue/liver\#img, (https://www.proteinatlas.org/ENSG00000178999AURKB/pathology/liver+cancer\#img); CDK1: (https://www.proteinatlas.org/ENSG00000170312-CDK1/ tissue/liver\#img), (https://www.proteinatlas.org/ENSG00000170312-CDK1/pathology/liver+cancer\#img); EHHADH: (https://www.proteinatlas.org/ ENSG00000113790-EHHADH/tissue\#img), (https:/www.proteinatlas.org/ENSG00000113790-EHHADH/pathology/liver+cancer\#img); CDC20: (https://www.proteinatlas.org/ENSG00000117399-CDC20/tissue/liver\#img), (https://www.proteinatlas.org/ENSG00000117399-CDC20/pathology/ liver+cancer\#img);CCNA2:(https://www.proteinatlas.org/ENSG00000145386-CCNA2/tissue/liver\#img),(https://www.proteinatlas.org/ENSG00000145386CCNA2/pathology/liver+cancer\#img; CCNB1: (https://www.proteinatlas.org/ENSG00000134057-CCNB1/tissue/liver\#img), (https://www. proteinatlas.org/ENSG00000134057-CCNB1/pathology/liver+cancer\#img);APOA1:(https://www.proteinatlas.org/ENSG00000118137-APOA1/tissue/liver\#img), (https:/www.proteinatlas.org/ENSG00000118137APOA1/pathology/liver+cancer\#img); UBB: (https://www.proteinatlas.org/ENSG00000170315-UBB/ tissue/liver\#img), (https://www.proteinatlas.org/ENSG00000170315-UBB/pathology/liver+cancer\#img). (C) IOD level of hub genes in immunohistochemistry sample images. ${ }^{*} \mathrm{P}<0.05 ;{ }^{* *} \mathrm{P}<0.01$ vs. normal tissues. IOD, integrated optical density; LIHC, liver hepatocellular carcinoma; AURKB, aurora kinase B; CDK1, cyclin-dependent kinase 1; EHHADH, enoyl-CoA hydratase and 3-hydroxyacyl CoA dehydrogenase; CDC20, cell division cycle 20; CCNA2/B1, cyclin A2/B1; APOA1, apolipoprotein A1; UBB, ubiquitin B.

signaling pathway' and the 'PPAR signaling pathway' (Fig. 4A). Correlation analysis in the cBioPortal database revealed that most of the hub genes were correlated with each other when their expression changes were in the same direction, such as 
A

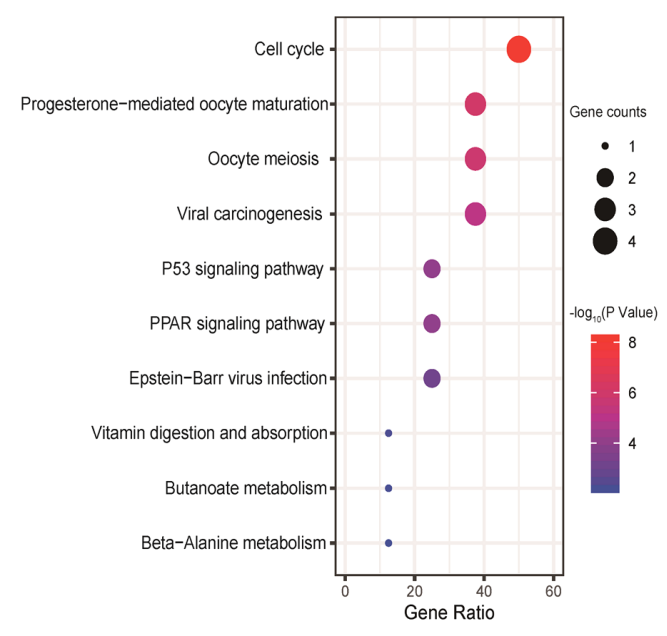

B

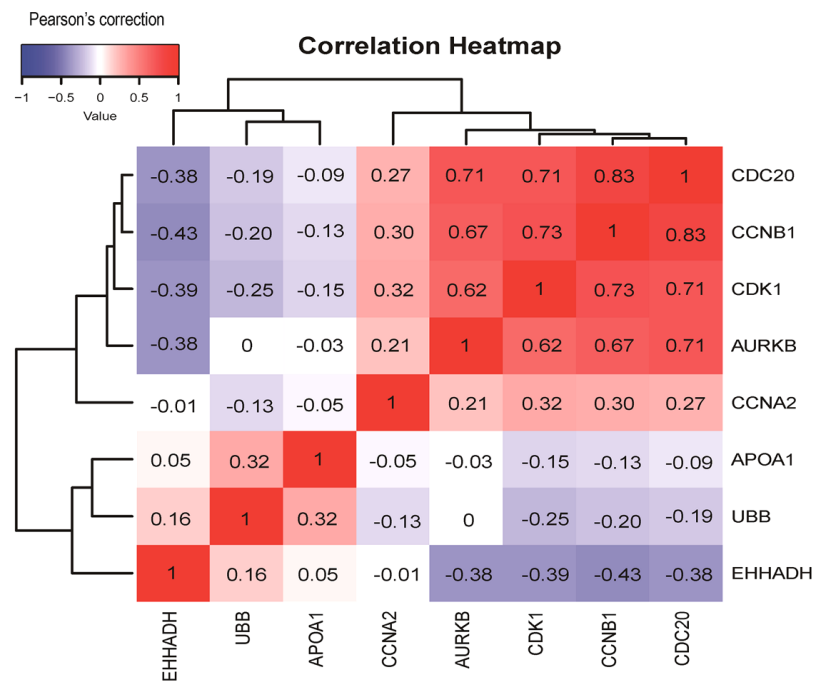

C

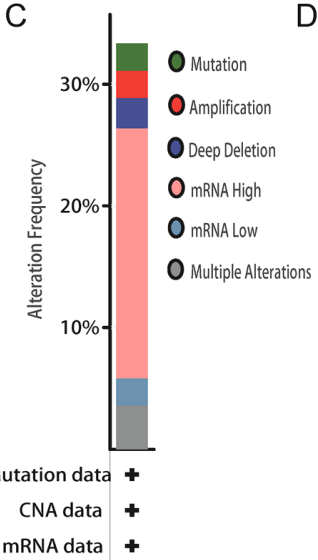

D coc20 \%

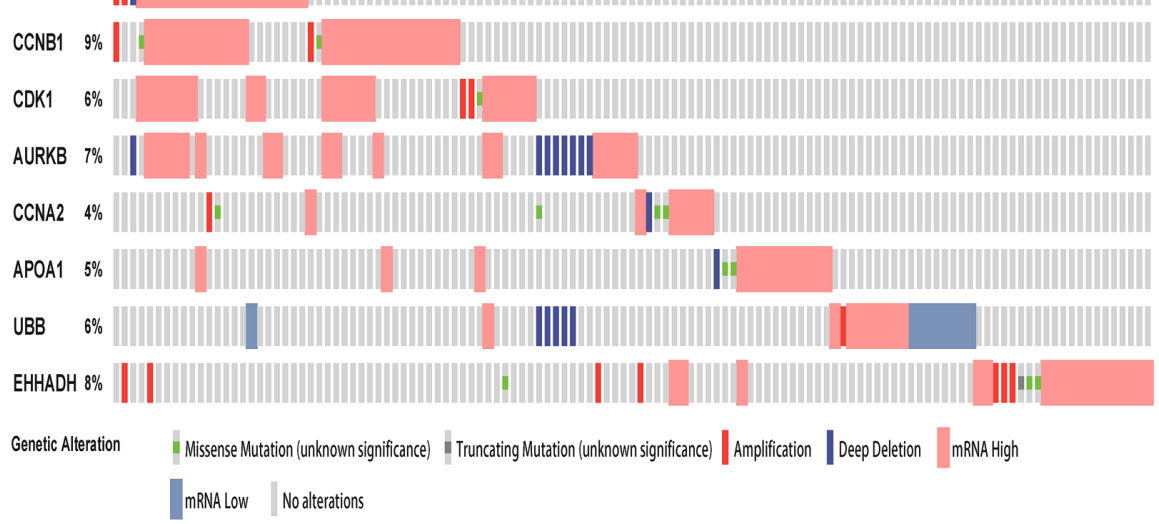

Figure 4. Pathway enrichment, correlation and mutation rate of hub genes. (A) Top 10 enriched pathways of hub genes. (B) Correlation heatmap. Blue indicates that there is a negative correlation between hub genes, red indicates that there is a positive correlation between hub genes and the number represents the Pearson's correlation coefficient. (C and D) Rate of genetic variation caused by abnormal changes of hub genes in tumor tissues. (C) The abnormal expression of the eight hub genes were found in $>30 \%$ of all samples. (D) Taking CDC20 as an example, the number of samples with gene CDC20 alterations in all samples accounted for $6 \%$ of the total number of samples. Green represents 'Missense Mutation'; dark gray represents 'Truncating Mutation'; red represents 'Amplification'; purple represents 'Deep Deletion'; flesh color represents 'mRNA High'; blue represents 'mRNA Low'; light gray represents 'No alterations'. AURKB, aurora kinase B; CDK1, cyclin-dependent kinase 1; EHHADH, enoyl-CoA hydratase and 3-hydroxyacyl CoA dehydrogenase; CDC20, cell division cycle 20; CCNA2/B1, cyclin A2/B1; APOA1, apolipoprotein A1; UBB, ubiquitin B.

CDC20 with CCNB1, CCNB1 with CDK1, and CDC20 with AURKB, which were highly and positively correlated with each other. By contrast, there were significant negative correlations between all downregulated hub genes and all upregulated hub genes, such as EHHADH with AURKB, CDK1, CCNB1 and CDC20 (Fig. 4B). According to the genetic changes of hub genes, it was revealed that the abnormal expression of the 8 hub genes were found in $\geq 30 \%$ of all samples (Fig. 4C and D).

Clinical significance of hub genes. Kaplan-Meier curves of the 8 hub genes revealed that the higher the expression levels of CDC20, CCNB1, AURKB, CCNA2 and CDK1, and the lower the expression levels of APOA1 and UBB, the poorer the OS, RFS, PFS and DSS of the patients (Fig. 5). In addition, low expression levels of EHHADH were associated with lower OS, DSS and PFS rates, while P-values for RFS was not statistically significant, as shown in Fig. 5. Based on UALCAN online database, for hub genes CDC20, CDK1, CCNB1, CCNA2 and AURKB, the higher their expression levels, the higher the stage in HCC tissues, while for APOAland EHHADH, the lower its expression levels, the higher the stage. However, it is worth noting that due to the small sample size of stage 4 , a certain bias in the results cannot be ruled out. Therefore, stage 4 is not included in the statistics. Besides, there was no significant relationship between UBB expression and tumor stage. (Fig. 6A). Furthermore, in the UALCAN online database, for hub genes CDC20, CDK1, CCNB1, CCNA2 and AURKB, the higher their expression levels, the higher the grade in HCC tissues, while for EHHADH, the lower its expression levels, the higher the grade. Besides, there was no significant relationship between APOA1 and UBB expression and tumor grade (Fig. 6B).

CMap analysis. CMap analysis was used to identify potential small molecular compounds that may reverse the altered expression levels of DEGs. The most five significant small molecular compounds were phenoxybenzamine, GW-8510, resveratrol, 0175029-0000 and daunorubicin (Table II). 

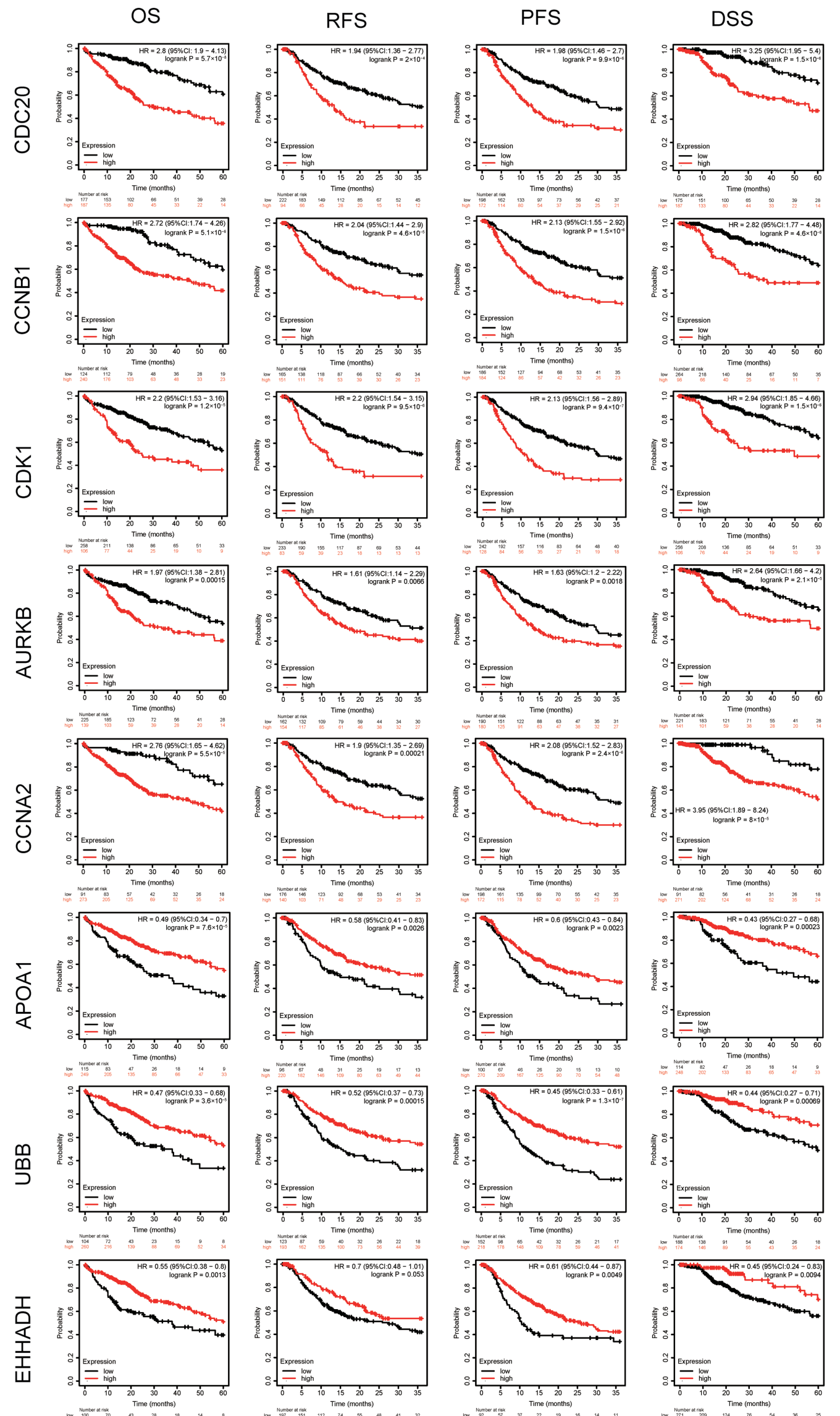

Figure 5. Survival analysis of hub genes. The higher the expression levels of CDC20, CCNB1, AURKB, CCNA2, CDK1 and the lower the expression levels of APOA1 and UBB, the poorer the OS, RFS, PFS and DSS of the patients. In addition, lower levels of EHHADH resulted in lower OS, DSS and PFS rates, but P-values for RFS were not statistically significant. AURKB, aurora kinase B; CDK1, cyclin-dependent kinase 1; EHHADH, enoyl-CoA hydratase and 3-hydroxyacyl CoA dehydrogenase; CDC20, cell division cycle 20; CCNA2/B1, cyclin A2/B1; APOA1, apolipoprotein A1; UBB, ubiquitin B; OS, overall survival; RFS, relapse-free survival; PFS, progression-free survival; DSS, disease-specific survival; HR, hazard ratio; CI, confidence interval. 


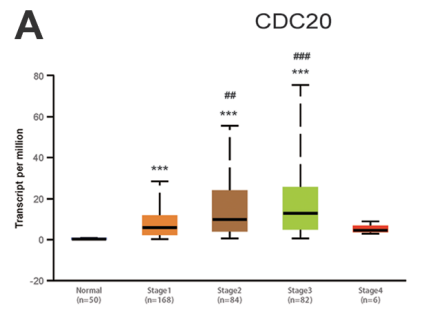

AURKB

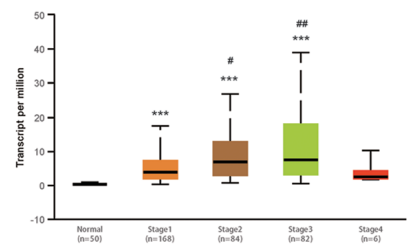

UBB

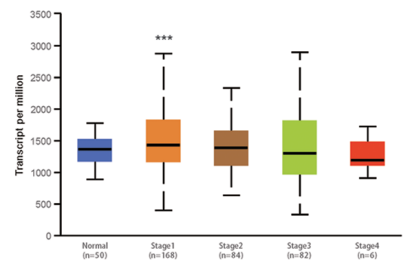

B

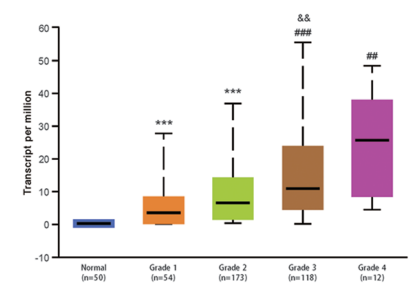

AURKB

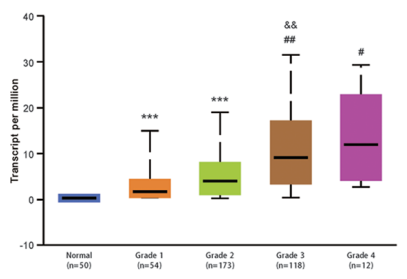

UBB

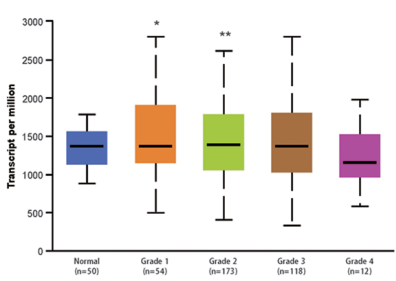

CCNB1

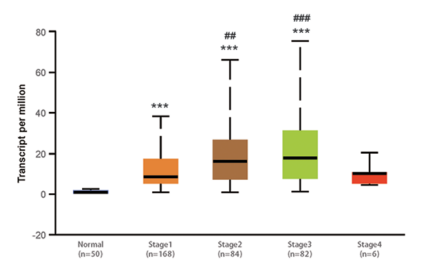

CCNA2

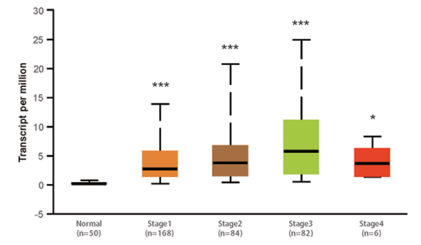

EHHADH

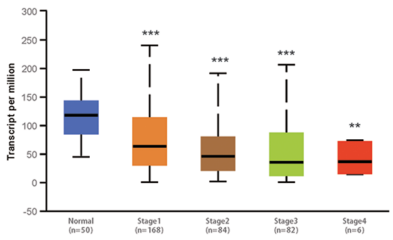

CCNB1

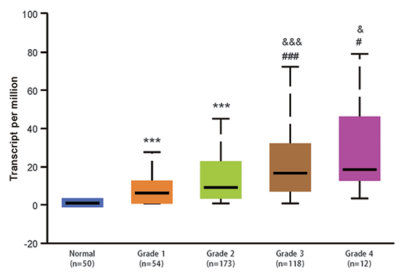

CCNA2

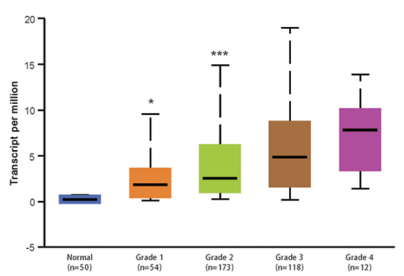

EHHADH

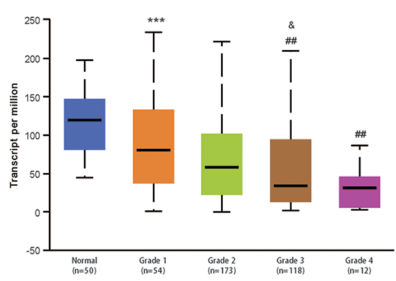

CDK1

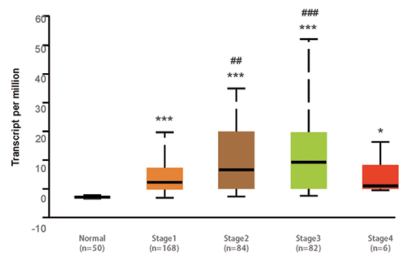

APOA1

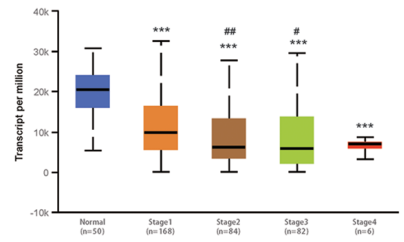

CDK1

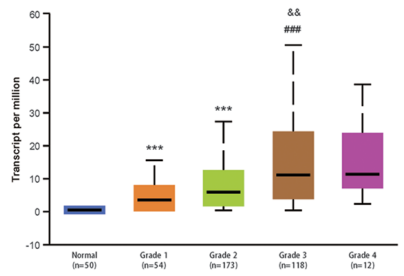

APOA1

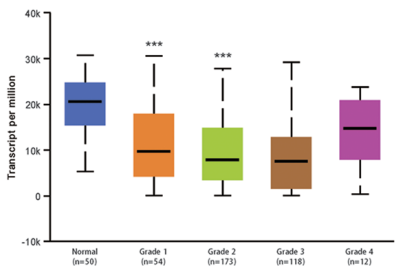

Figure 6. Association between hub genes and pathological stage and grade. (A) For hub genes CDC20, CDK1, CCNB1, CCNA2 and AURKB, the higher their expression levels, the higher the stage in HCC tissues, while for APOAland EHHADH, the lower its expression levels, the higher the stage (Due to the small sample size of stage 4, a certain bias in the results cannot be ruled out, therefore, stage 4 is not included in the statistics). Besides, there was no significant relationship between UBB expression and tumor stage. (B) For hub genes CDC20, CCNB1, CDK1 and AURKB, the higher their expression levels, the higher the grade and therefore the lower the differentiation level in HCC tissues. For EHHADH, the lower its expression levels, the higher the grade and the lower the differentiation level in HCC tissues. For the hub gene CCNA2, the higher the level of CCNA2 expression in liver cancer tissues, the lower the differentiation level was, but the difference was not statistically significant. Comparisons between grade $1 / 2$ and $3 / 4$ for APOA1 and UBB revealed no statistical significance. ${ }^{*} \mathrm{P}<0.05,{ }^{* *} \mathrm{P}<0.01$ and ${ }^{* * *} \mathrm{P}<0.001$ vs. normal; ${ }^{\#} \mathrm{P}<0.05,{ }^{\# \#} \mathrm{P}<0.01$ and ${ }^{\# \# \#} \mathrm{P}<0.001$ vs. Grade 1 or Stage $1 ;{ }^{\&} \mathrm{P}<0.05$, ${ }^{\& \&} \mathrm{P}<0.01$ and ${ }^{\& \& \&} \mathrm{P}<0.001$ vs. Grade 2 . AURKB, aurora kinase B; CDK1, cyclin-dependent kinase 1; EHHADH, enoyl-CoA hydratase and 3-hydroxyacyl CoA dehydrogenase; CDC20, cell division cycle 20; CCNA2/B1, cyclin A2/B1; APOA1, apolipoprotein A1; UBB, ubiquitin B; HCC, hepatocellular carcinoma; TMP, transcripts per million; Pr(>F), P-value. 
Table II. CMap analysis of the 20 most significant small molecular compounds that may reverse the altered expression levels of the differentially expressed genes.

\begin{tabular}{|c|c|c|c|c|}
\hline CMap name & Mean & Enrichment & P-value & Percent non-null \\
\hline Phenoxybenzamine & -0.873 & -0.995 & $<0.00001$ & 100 \\
\hline GW-8510 & -0.799 & -0.952 & $<0.00001$ & 100 \\
\hline Resveratrol & -0.781 & -0.827 & $<0.00001$ & 100 \\
\hline 0175029-0000 & -0.777 & -0.887 & $<0.00001$ & 100 \\
\hline Daunorubicin & -0.756 & -0.944 & $<0.00001$ & 100 \\
\hline Apigenin & -0.792 & -0.927 & 0.00002 & 100 \\
\hline Camptothecin & -0.778 & -0.980 & 0.00004 & 100 \\
\hline Thioguanosine & -0.812 & -0.898 & 0.00022 & 100 \\
\hline Irinotecan & -0.763 & -0.953 & 0.00022 & 100 \\
\hline Chrysin & -0.733 & -0.954 & 0.00022 & 100 \\
\hline Luteolin & -0.703 & -0.893 & 0.00026 & 100 \\
\hline Trifluridine & -0.729 & -0.849 & 0.00095 & 100 \\
\hline Alsterpaullone & -0.753 & -0.905 & 0.00162 & 100 \\
\hline Doxorubicin & -0.744 & -0.877 & 0.00375 & 100 \\
\hline Thiostrepton & -0.713 & -0.779 & 0.00493 & 100 \\
\hline Menadione & -0.766 & -0.950 & 0.00545 & 100 \\
\hline Monobenzone & -0.730 & -0.753 & 0.00758 & 100 \\
\hline Ms-275 & -0.729 & -0.931 & 0.01006 & 100 \\
\hline Azacitidine & -0.719 & -0.798 & 0.01683 & 100 \\
\hline 8-azaguanine & -0.753 & -0.683 & 0.02216 & 100 \\
\hline
\end{tabular}

CMap, Connectivity Map.

\section{Discussion}

Primary liver cancer is a rapidly progressing malignant tumor, and early diagnosis and treatment are of great importance for patients. Although great progress has been made, the diagnostic and prognostic biomarkers, and targeted agents of HCC have not been fully revealed. In the present study, next-generation high-throughput sequencing technology, namely RNA-seq, was applied to provide robust raw data for further exploring the critical genes and pathways associated with HBV-associated early stage HCC. CMap analysis was applied to identify promising targeted agents against $\mathrm{HBV}$-associated early stage HCC.

In the present study, a total of 1,582 DEGs were identified. KEGG enrichment analysis of DEGs revealed that the DEGs were mostly enriched in metabolic pathways, which was consistent with previous results showing that the dysfunction of metabolic pathways was a critical risk factor for HCC, especially for those infected with HBV or HCV (32-34). 'Fatty acid degradation' and 'PPAR signaling pathway' in KEGG analysis, and 'steroid metabolic process' and 'fatty acid beta-oxidation' in GO BP analysis, are all strongly associated with fat metabolism (35), suggesting that fat metabolism disorders may be closely associated with HCC. Yamashita et al (36) revealed that the lipogenic pathway is activated in HCC and can promote HCC proliferation and may have close association with high mortality. Jiang et al (18) demonstrated that when cholesterol homeostasis was impaired, HBV-infected patients with HCC had the lowest overall survival rate and a worse prognosis after the initial surgery treatment. In the present study, the hub gene EHHADH, which is involved in fatty acid metabolism, was enriched in pathways such as 'fatty acid metabolism', 'fatty acid beta-oxidation' and the 'PPAR signaling pathway'; the hub gene APOA1, which is associated with tumor metabolism, was also enriched in the 'PPAR signaling pathway'.

Additionally, GO analysis of BP provided evidence that the DEGs were significantly enriched in the 'oxidation-reduction process' (redox). To the best of our knowledge, no reports have confirmed an association between redox and HCC, but there are studies about oxidative stress and HCC. It has been demonstrated that oxidative stress can lead to unrepaired DNA damage, especially of nuclear and mitochondrial DNA, which may contribute to modifications and mutations of gene expression, and may subsequently induce the development of HCC $(37,38)$. Additionally, KEGG analysis of the hub genes in the present study revealed that the 'p53 signaling pathway' was an important pathway. Previous studies have reported that the p53 signaling pathway affects the proliferation, metastasis and radiotherapy sensitivity of $\operatorname{HCC}(39,40)$, and is associated with redox (41-43). Under physiological conditions, cells maintain a balance of redox reactions by generating and removing reactive oxygen species (ROS) and reactive nitrogen species (RNS) (44). If the steady state of the redox reaction is altered, it will induce oxidative stress, which in turn can lead to excessive production of ROS and RNS (44). Both mild and severe oxidative stress will activate $\mathrm{p} 53$, which will activate different target genes, thus determining the different fate of the cell $(37,44-46)$. Under low levels of oxidative stress, 
activated p53 serves an anti-oxidant role, reduces the levels of ROS, restores the balance of redox reactions and enables cells to survive, while under high levels of oxidative stress, p53 serves a pro-oxidative role, exacerbating oxidative stress and leading to cell cycle arrest and apoptosis (37,44-46). ROS generated under oxidative stress serves an important regulatory role in the occurrence and development of tumors. Increased ROS production can induce mutations of tumor suppressor genes, such as p53, and genetic instability (44). Loss of p53 function will increase the production of ROS, so that cells gradually acquire the ability to continuously proliferate and invade, which finally leads to the occurrence and development of cancer $(44,47)$. Maurya and Vinayak $(48)$ revealed that in HepG2 cells, oxidative stress reduces p53 levels, promotes cell proliferation and reduces apoptosis by regulating the expression of its target genes. Additionally, it has been demonstrated that p53 transcription factor is sensitive to redox and may be upregulated when the DNA is injured by oxidative stress (37). Consistently, the hub genes CDK1 and CCNB1, which were enriched in the p53 signaling pathway, were upregulated in the present analysis.

In the present study, GO analysis of BP indicated that the DEGs were associated with 'sister chromatid cohesion', and KEGG analysis provided evidence that the DEGs and hub genes were all enriched in the 'cell cycle' and the 'p53 signaling pathway'. These results are consistent with the previous findings that cell life and death are associated with the cell cycle, and the disruption of the cell cycle may lead to cell cycle arrest, dysfunction of transcription and uncontrolled cell growth, which can be the fundament of tumorigenesis and can affect the prognosis of cancer $(37,49,50)$. p53 is an important tumor suppressor. p53 and its target genes constitute a complex p53 signaling pathway that regulates various biological functions (39). In the case of DNA damage and oncogene activation, p53 can serve a role in cell cycle checkpoints to regulate cell cycle arrest, apoptosis and aging to maintain gene integrity and prevent tumor development (51). The alternation of p53 and/or its target genes makes the p53 signaling pathway lose its role of inhibiting tumorigenesis and obtain carcinogenic functions, such as promoting the proliferation, metastasis, anti-apoptosis ability and angiogenesis of tumor cells $(39,51,52)$. Hub genes such as CDK1 (53), CDC20 (54), CCNB1 (55) and CCNA2 (56), which have been associated with cell cycle-mediated proliferation and invasion of HCC cells, were all enriched in the 'cell cycle' process, and CDK1 and CCNB1 were enriched in the 'p53 signaling pathway' in the present study.

Additionally, 'viral carcinogenesis' and the 'PPAR signaling pathway' are involved in viral infection $(57,58)$, which is consistent with the present results in the KEGG enrichment analysis of DEGs and hub genes of HBV-associated early stage HCC. Overall, the dysfunction of metabolic pathways (especially disorder of fat metabolism), the cell cycle, oxidation-reduction process and viral carcinogenesis may serve a critical role in the carcinogenesis of HBV-associated early stage HCC and may be a potential therapeutic target.

A total of 8 hub genes were identified in the present study, among which AURKB, CDK1, CCNA2, CDC20 and CCNB1 were upregulated, while EHHADH, APOA1 and UBB were downregulated. The hub genes CDK1, CCNA2, CDC20 and
CCNB1 are closely associated with the cell cycle and are closely connected with each other; for instance, CCNA2 can activate CDK1 (59), and they can promote the formation of $\mathrm{HCC}$ in combination with other genes (60). Previous studies have demonstrated that poor clinicopathological characteristics and recurrence after surgery are closely associated with AURKB upregulation, which may be used as an independent prognostic predictor and a target for adjuvant therapy of HCC (61-63). Wu et al (64) revealed that the levels of CDK1 are higher in patients with HCC compared with healthy individuals, which is in accordance with the present study. Furthermore, Ito et al (53) reported that CDK1 serves an important regulatory role in the $\mathrm{G}_{2} / \mathrm{M}$ phase of the cell cycle and the proliferation of HCC cells, and is directly associated with portal vein invasion, distant metastasis and low differentiation. Increasing numbers of studies have demonstrated that the expression levels of AURKB, CDK1, CDC20, CCNB1 and CCNA2 in HCC are higher than those in normal tissues $(54,65)$, which is in accordance with the present study. Moreover, in the present study, high expression levels of these genes were associated with poorer OS, RFS, PFS and DSS rates, indicating that higher levels of these genes may predict a higher risk of recurrence and metastasis of HCC after surgery, and a poorer prognosis. Therefore, active monitoring and adjuvant therapy should be provided to patients with HCC. In addition, the higher the expression levels of CDC20, CDK1, CCNB1and AURKB, the higher the grade of HCC cells and the worse the prognosis. Therefore, these genes may be used for diagnosing the occurrence of $\mathrm{HBV}$-associated early stage $\mathrm{HCC}$, as well as for predicting the prognosis of patients and potentially acting as therapeutic targets.

Among the three downregulated hub genes (EHHADH, APOA1 and UBB), it has been reported that EHHADH can encode an enzyme with two functions, which can regulate the metabolism of fatty acids and nitrogen (66). Previous studies have demonstrated that APOA1 can inhibit the progression and metastasis of various types of tumor, such as breast and ovarian cancer $(67,68)$. APOA1 does not directly inhibit tumor growth or promote apoptosis of tumor cells, but acts via indirect effects. On one hand, the APOA1 mimetic peptide can inhibit the signaling pathway of tumor vascular epithelial growth factor and basic fibroblast growth factor, therefore inhibiting tumor-associated angiogenesis (69); on the other hand, Zamanian-Daryoush et al (70) revealed that APOA1 can effectively regulate autogenic and acquired immune responses, reduce the microenvironment that allows tumor growth, and increase antitumor macrophages and CD8 T cells, thereby indirectly inhibiting tumor growth and metabolism.

Based on the UALCAN databases, the present study revealed that low EHHADH expression was associated with a high grade of HCC cells, corresponding to a poor prognosis. Additionally, lower levels of APOA1 were associated with lower OS, PFS, RFS and DSS rates, while lower levels of EHHADH were associated with lower OS, DSS and PFS rates. These results indicated that patients with $\mathrm{HCC}$ with low EHHADH and APOA1 expression tended to have rapid tumor growth after surgery, and higher mortality compared with those with high EHHADH and APOA1 expression, suggesting that EHHADH and APOA1 may be tumor suppressor genes. 
There are different results among previous studies regarding UBB. Some studies have demonstrated that UBB-knockout can effectively downregulate ubiquitin and inhibit the growth of tumors (71), such as non-small cell lung cancer (72). Additionally, Tian et al (73) reported that UBB serves an important regulatory role in maintaining the characteristics of cancer stem cells and the initiation of cervical cancer. However, a retrospective study conducted by Valdagni et al (74) revealed that patients with prostate cancer who underwent radiotherapy had a lower risk of rectal bleeding when genes such as UBB were significantly downregulated. It can be seen that UBB may have different roles in the diagnosis and treatment of different types of cancer; however, no studies have yet clarified the role of UBB in HCC.

The present study revealed that UBB downregulation was closely associated with poor OS, RFS, PFS and DSS rates, suggesting that in patients with $\mathrm{HBV}$-associated early stage HCC with low UBB expression, the tumor may be prone to relapse and rapid growth after surgery, and patients have a high mortality rate. Therefore, patients should be closely monitored after surgery and treated swiftly if abnormalities are detected, hoping to improve their prognosis. Additionally, it could be inferred that UBB may be a tumor suppressor gene.

In the present study, 20 small molecular compounds were identified based on the analysis of DEGs, which may be able to reverse the altered expression of the DEGs; therefore, they may be promising targeted agents against $\mathrm{HBV}$-associated early stage HCC. Among them, daunorubicin, irinotecan and doxorubicin have already been reported to have effectiveness in clinical trials (75-77). Additionally, resveratrol, apigenin, luteolin and menadione have been demonstrated to display good therapeutic effects in animal models (45,78-80). Camptothecin, chrysin and MS-275 have only been studied in vivo or in cell experiments, but may have potential to treat HCC (81-83). Other small molecular compounds have not yet been demonstrated to have effectiveness against HCC, and therefore further research is required.

Previous studies have revealed that some of these compounds act on important signaling pathways that have been identified in the present study, which further supports our views on the mechanism of HBV-associated HCC formation. Lin et al (78) reported that resveratrol can promote the recovery of the fatty liver and effectively decrease the incidence of HCC caused by the HBV X protein (HBX) in HBX transgenic mice via downregulating lipogenesis, promoting transient liver regeneration and stimulating antioxidant activity. Additionally, it has been demonstrated that resveratrol has therapeutic effects on HCC through the p53 (84), cell cycle (85-87). A previous study revealed that menadione kills tumor cells by influencing their redox cycle (45). In addition, other small molecular compounds that act on the cell cycle are apigenin, luteolin and doxorubicin, while further small molecular compounds that are associated with the p53 pathway are irinotecan and doxorubicin (89-93). Given the broad spectrum of these small molecular compounds, it is not surprising that these compounds may act as promising therapeutic targets for HBV-associated early stage HCC.

In conclusion, the present study revealed eight hub genes, important signaling pathways and potential targeted agents based on the high-throughput sequencing method. These may provide clues for revealing the molecular mechanism of early stage HCC caused by HBV infection and offer potential therapeutic, diagnostic and prognostic biomarkers. Furthermore, these compounds may represent promising targeted therapeutic agents against HBV-associated early stage HCC. However, in order to verify the predictions of the present study, further research is required.

\section{Acknowledgements}

Not applicable.

\section{Funding}

The present study was supported by the High-level University Construction of Guangzhou University of Chinese Medicine (grant no. A1-AFD018181A29) and Project of Administration of Traditional Chinese Medicine of Guangdong Province of China (Project No. 20201109).

\section{Availability of data and materials}

The datasets generated and/or analyzed during the current study are available in the GSE124535 dataset (https://www. ncbi.nlm.nih.gov/geo/query/acc.cgi?acc=GSE124535) from the Gene Expression Omnibus (GEO) database (https://www. ncbi.nlm.nih.gov/geo/).

\section{Authors' contributions}

ZZ,ZC and YT conceived and designed the study. ZZ performed the bioinformatics analysis. All authors contributed to data collection and analysis. ZZ, YT and ZC contributed to data visualization. $\mathrm{ZZ}$ wrote the manuscript. $\mathrm{ZC}$ and $\mathrm{YT}$ reviewed and edited the manuscript. All authors contributed to the manuscript and agreed to be responsible for all aspects of the work.

\section{Ethics approval and consent to participate}

Not applicable.

\section{Patient consent for publication}

Not applicable.

\section{Competing interests}

The authors declare that they have no competing interests.

\section{References}

1. Yang JD, Hainaut P, Gores GJ, Amadou A, Plymoth A and Roberts LR: A global view of hepatocellular carcinoma: Trends, risk, prevention and management. Nat Rev Gastroenterol Hepatol 16: 589-604, 2019.

2. Villanueva A: Hepatocellular carcinoma. N Engl J Med 380: 1450-1462, 2019

3. El-Serag HB and Rudolph KL: Hepatocellular carcinoma: Epidemiology and molecular carcinogenesis.Gastroenterology 132: 2557-2576, 2007.

4. Yang JD and Roberts LR: Hepatocellular carcinoma: A global view. Nat Rev Gastroenterol Hepatol 7: 448-458, 2010. 
5. Bruix J and Sherman M; Practice Guidelines Committee, American Association for the Study of Liver Diseases: Management of hepatocellular carcinoma. Hepatology 42: 1208-1236, 2005.

6. Vogel A, Cervantes A, Chau I, Daniele B, Llovet JM, Meyer T, Nault JC, Neumann U, Ricke J, Sangro B, et al: Hepatocellular carcinoma: ESMO clinical practice guidelines for diagnosis, treatment and follow-up. Ann Oncol 29 (Suppl 4): iv238-iv255, 2018

7. Bruix J and Sherman M; American Association for the Study of Liver Diseases: Management of hepatocellular carcinoma: An update. Hepatology 53: 1020-1022, 2011.

8. Varela M, Reig M, de la Mata M, Matilla A, Bustamante J, Pascual S, Turnes J, Aracil C, Del Val A, Pascasio JM, et al: Treatment approach of hepatocellular carcinoma in Spain Analysis of 705 patients from 62 centers. Med Clin (Barc) 134 569-576, 2010 (In Spanish)

9. Bargellini I, Sacco R, Bozzi E, Bertini M, Ginanni B, Romano A Cicorelli A, Tumino E, Federici G, Cioni R, et al: Transarterial chemoembolization in very early and early-stage hepatocellular carcinoma patients excluded from curative treatment: A prospective cohort study. Eur J Radiol 81: 1173-1178, 2012.

10. Song YG, Shin SW, Cho SK, Choi D, Rhim H, Lee MW, Kim YS, Park KB, Park HS, Choo SW, et al: Transarterial chemoembolization as first-line therapy for hepatocellular carcinomas infeasible for ultrasound-guided radiofrequency ablation: A retrospective cohort study of 116 patients. Acta Radiol 56: 70-77, 2015.

11. Kim JW, Kim JH, Sung KB, Ko HK, Shin JH, Kim PN, Choi HK Ko GY, Yoon HK, Chun SY and Gwon DI: Transarterial chemoembolization vs. radiofrequency ablation for the treatment of single hepatocellular carcinoma $2 \mathrm{~cm}$ or smaller. Am J Gastroenterol 109: 1234-1240, 2014.

12. Gopal P, Yopp AC, Waljee AK, Chiang J, Nehra M, Kandunoori P and Singal AG: Factors that affect accuracy of $\alpha$-fetoprotein test in detection of hepatocellular carcinoma in patients with cirrhosis. Clin Gastroenterol Hepatol 12: 870-877, 2014.

13. Chang TS, Wu YC, Tung SY, Wei KL, Hsieh YY, Huang HC, Chen WM, Shen CH, Lu CH, Wu CS, et al: Alpha-fetoprotein measurement benefits hepatocellular carcinoma surveillance in patients with cirrhosis. Am J Gastroenterol 110: 836-845, 2015.

14. Zhou J, Sun HC, Wang Z, Cong WM, Wang JH, Zeng MS, Yang JM, Bie P, Liu LX, Wen TF, et al: Guidelines for diagnosis and treatment of primary liver cancer in China (2017 edition). Liver Cancer 7: 235-260, 2018

15. Takikawa Y and Suzuki K: Is AFP a new reliable marker of liver regeneration in acute hepatic failure? J Gastroenterol 37: $681-682,2002$

16. Minguez B and Lachenmayer A: Diagnostic and prognostic molecular markers in hepatocellular carcinoma. Dis Markers 31: 181-190, 2011.

17. Wang Z, Gerstein M and Snyder M: RNA-Seq: A revolutionary tool for transcriptomics. Nat Rev Genet 10: 57-63, 2009.

18. Jiang Y, Sun A, Zhao Y, Ying W, Sun H, Yang X, Xing B, Sun W, Ren L, Hu B, et al: Proteomics identifies new therapeutic targets of early-stage hepatocellular carcinoma. Nature 567: 257-261, 2019.

19. Anders S, Pyl PT and Huber W: HTSeq-a python framework to work with high-throughput sequencing data. Bioinformatics 31 : 166-169, 2015.

20. Ritchie ME, Phipson B, Wu D, Hu Y, Law CW, Shi W and Smyth GK: limma powers differential expression analyses for RNA-sequencing and microarray studies. Nucleic Acids Res 43 : e47, 2015.

21. Huang da W, Sherman BT and Lempicki RA: Systematic and integrative analysis of large gene lists using DAVID bioinformatics resources. Nat Protoc 4: 44-57, 2009.

22. Xie C, Mao X, Huang J, Ding Y, Wu J, Dong S, Kong L, Gao G Li CY and Wei L: KOBAS 2.0: A web server for annotation and identification of enriched pathways and diseases. Nucleic Acids Res 39: W316-W322, 2011.

23. Szklarczyk D, Morris JH, Cook H, Kuhn M, Wyder S Simonovic M, Santos A, Doncheva NT, Roth A, Bork P, et al: The STRING database in 2017: Quality-controlled protein-protein association networks, made broadly accessible. Nucleic Acids Res 45: D362-D368, 2017.

24. Shannon P, Markiel A, Ozier O, Baliga NS, Wang JT, Ramage D, Amin N, Schwikowski B and Ideker T: Cytoscape: A software environment for integrated models of biomolecular interaction networks. Genome Res 13: 2498-2504, 2003

25. Chin $\mathrm{CH}$, Chen $\mathrm{SH}, \mathrm{Wu} \mathrm{HH}, \mathrm{Ho} \mathrm{CW}$, Ko MT and Lin CY: cytoHubba: Identifying hub objects and sub-networks from complex interactome. BMC Syst Biol 8 (Suppl 4): S11, 2014.
26. Bringmann LF, Elmer T, Epskamp S, Krause RW, Schoch D, Wichers M, Wigman JTW and Snippe E: What do centrality measures measure in psychological networks? J Abnorm Psychol 128: 892-903, 2019

27. Chandrashekar DS, Bashel B, Balasubramanya SAH, Creighton CJ, Ponce-Rodriguez I, Chakravarthi BVSK and Varambally S: UALCAN: A portal for facilitating tumor subgroup gene expression and survival analyses. Neoplasia 19: 649-658, 2017.

28. Uhlén M, Fagerberg L, Hallström BM, Lindskog C, Oksvold P, Mardinoglu A, Sivertsson Å, Kampf C, Sjöstedt E, Asplund A, et al: Proteomics. Tissue-based map of the human proteome. Science 347: 1260419, 2015.

29. Cerami E, Gao J, Dogrusoz U, Gross BE, Sumer SO, Aksoy BA, Jacobsen A, Byrne CJ, Heuer ML, Larsson E, et al: The cBio cancer genomics portal: An open platform for exploring multidimensional cancer genomics data. Cancer Discov 2: 401-404, 2012

30. Nagy Á, Lánczky A, Menyhárt O and Győrffy B: Validation of miRNA prognostic power in hepatocellular carcinoma using expression data of independent datasets. Sci Rep 8: 9227, 2018.

31. Subramanian A, Narayan R, Corsello SM, Peck DD, Natoli TE, Lu X, Gould J, Davis JF, Tubelli AA, Asiedu JK, et al: A next generation connectivity map: L1000 platform and the first 1,000,000 profiles. Cell 171: 1437-1452 e17, 2017.

32. Fu SC, Huang YW, Wang TC, Hu JT, Chen DS and Yang SS: Increased risk of hepatocellular carcinoma in chronic hepatitis B patients with new onset diabetes: A nationwide cohort study. Aliment Pharmacol Ther 41: 1200-1209, 2015.

33. Huang YW, Wang TC, Yang SS, Lin SY, Fu SC, Hu JT, Liu CJ, Kao JH and Chen DS: Increased risk of hepatocellular carcinoma in chronic hepatitis $C$ patients with new onset diabetes: A nation-wide cohort study. Aliment Pharmacol Ther 42: 902-911, 2015.

34. Xia H, Chen J, Sekar K, Shi M, Xie T and Hui KM: Clinical and metabolomics analysis of hepatocellular carcinoma patients with diabetes mellitus. Metabolomics 15: 156, 2019.

35. Zhou QC, Shi B, Jiao LF, Jin M, Sun P, Ding LY and Yuan Y: Hepatopancreas and ovarian transcriptome response to different dietary soybean lecithin levels in portunus trituberculatus. Comp Biochem Physiol Part D Genomics Proteomics 31: 100600, 2019.

36. Yamashita T, Honda M, Takatori $H$, Nishino $R$, Minato $H$, Takamura H, Ohta T and Kaneko S: Activation of lipogenic pathway correlates with cell proliferation and poor prognosis in hepatocellular carcinoma. J Hepatol 50: 100-110, 2009.

37. Li N, Li L and Chen Y: The identification of core gene expression signature in hepatocellular carcinoma. Oxid Med Cell Longev 2018: 3478305, 2018.

38. Zhong H, Xiao M, Zarkovic K, Zhu M, Sa R, Lu J, Tao Y, Chen Q, Xia L, Cheng S, et al: Mitochondrial control of apoptosis through modulation of cardiolipin oxidation in hepatocellular carcinoma: A novel link between oxidative stress and cancer. Free Radic Biol Med 102: 67-76, 2017.

39. Liu J, Zhang C and Feng Z: Tumor suppressor p53 and its gain-of-function mutants in cancer. Acta Biochim Biophys Sin (Shanghai) 46: 170-179, 2014.

40. Rebouissou S and Nault JC: Advances in molecular classification and precision oncology in hepatocellular carcinoma. J Hepatol 72: 215-229, 2020.

41. Shao Y, Song X, Jiang W, Chen Y, Ning Z, Gu W and Jiang J: MicroRNA-621 acts as a tumor radiosensitizer by directly targeting SETDB1 in hepatocellular carcinoma. Mol Ther 27: 355-364, 2019.

42. Pan YH, Yang M, Liu LP, Wu DC, Li MY and Su SG: UBE2S enhances the ubiquitination of p53 and exerts oncogenic activities in hepatocellular carcinoma. Biochem Biophys Res Commun 503: 895-902, 2018.

43. Sun G, Sui X, Han D, Gao J, Liu Y and Zhou L: TRIM59 promotes cell proliferation, migration and invasion in human hepatocellular carcinoma cells. Pharmazie 72: 674-679, 2017.

44. Trachootham D, Lu W, Ogasawara MA, Nilsa RD and Huang P Redox regulation of cell survival. Antioxid Redox Signal 10: 1343-1374, 2008

45. Oztopcu-Vatan P, Sayitoglu M, Gunindi M and Inan E: Cytotoxic and apoptotic effects of menadione on rat hepatocellular carcinoma cells. Cy totechnology 67: 1003-1009, 2015.

46. Liu D and Xu Y: p53, oxidative stress, and aging. Antioxid Redox Signal 15: 1669-1678, 2011

47. D'Souza LC, Mishra S, Chakraborty A, Shekher A, Sharma A and GuptaSC: Oxidative stress and cancerdevelopment: Are noncoding RNAs the missing links? Antioxid Redox Signal: Jan 24, 2020 (Epub ahead of print). doi: 10.1089/ars.2019.7987. 
48. Maurya AK and Vinayak M: Anticarcinogenic action of quercetin by downregulation of phosphatidylinositol 3-kinase (PI3K) and protein kinase $\mathrm{C}$ (PKC) via induction of p53 in hepatocellular carcinoma (HepG2) cell line. Mol Biol Rep 42: 1419-1429, 2015.

49. Liu S, Yang TB, Nan YL, Li AH, Pan DX, Xu Y, Li S, Li T, Zeng XY and Qiu XQ: Genetic variants of cell cycle pathway genes predict disease-free survival of hepatocellular carcinoma. Cancer Med 6: 1512-1522, 2017.

50. Maddika S, Ande SR, Panigrahi S, Paranjothy T, Weglarczyk K, Zuse A, Eshraghi M, Manda KD, Wiechec E and Los M: Cell survival, cell death and cell cycle pathways are interconnected: Implications for cancer therapy. Drug Resist Updat 10: 13-29, 2007.

51. Giono LE and Manfredi JJ: The p53 tumor suppressor participates in multiple cell cycle checkpoints. J Cell Physiol 209: 13-20, 2006

52. Chen J: The cell-cycle arrest and apoptotic functions of p53 in tumor initiation and progression. Cold Spring Harb Perspect Med 6: a026104, 2016.

53. Ito $Y$, Takeda T, Sakon M, Monden M, Tsujimoto $M$ and Matsuura N: Expression and prognostic role of cyclin-dependent kinase $1(\mathrm{cdc} 2)$ in hepatocellular carcinoma. Oncology 59: 68-74, 2000.

54. Li J, Gao JZ, Du JL, Huang ZX and Wei LX: Increased CDC20 expression is associated with development and progression of hepatocellular carcinoma. Int J Oncol 45: 1547-1555, 2014.

55. Gu J, Liu X, Li J and He Y: MicroRNA-144 inhibits cell proliferation, migration and invasion in human hepatocellular carcinoma by targeting CCNB1. Cancer Cell Int 19: 15, 2019.

56. Bayard Q, Meunier L, Peneau C, Renault V, Shinde J, Nault JC Mami I, Couchy G, Amaddeo G, Tubacher E, et al: Cyclin A2/E1 activation defines a hepatocellular carcinoma subclass with a rearrangement signature of replication stress. Nat Commun 9 : $5235,2018$.

57. Shi Y, Li Y, Huang C, Ying L, Xue J, Wu H, Chen Z and Yang Z: Resveratrol enhances HBV replication through activating Sirt1-PGC-1 $\alpha$-PPAR $\alpha$ pathway. Sci Rep 6: 24744, 2016.

58. Huang JY, Chou SF, Lee JW, Chen HL, Chen CM, Tao MH and Shih C: MicroRNA-130a can inhibit hepatitis B virus replication via targeting PGC1 $\alpha$ and PPAR $\gamma$. RNA 21: 385-400, 2015.

59. Kanakkanthara A, Jeganathan KB, Limzerwala JF, Baker DJ, Hamada M, Nam HJ, van Deursen WH, Hamada N, Naylor RM, Becker NA, et al: Cyclin A2 is an RNA binding protein that controls Mre11 mRNA translation. Science 353: 1549-1552, 2016.

60. Yan H,Li Z, Shen Q, Wang Q, Tian J, Jiang Q and Gao L: Aberrant expression of cell cycle and material metabolism related genes contributes to hepatocellular carcinoma occurrence. Pathol Res Pract 213: 316-321, 2017.

61. Lin ZZ, Jeng YM, Hu FC, Pan HW, Tsao HW, Lai PL, Lee PH, Cheng AL and Hsu HC: Significance of Aurora B overexpression in hepatocellular carcinoma. Aurora B overexpression in HCC. BMC Cancer 10: 461, 2010.

62. Yasen M, Mizushima H, Mogushi K, Obulhasim G, Miyaguchi K, Inoue K, Nakahara I, Ohta T, Aihara A, Tanaka S, et al: Expression of Aurora B and alternative variant forms in hepatocellular carcinoma and adjacent tissue. Cancer Sci 100: 472-480, 2009.

63. Tanaka S, Arii S, Yasen M, Mogushi K, Su NT, Zhao C, Imoto I, Eishi Y, Inazawa J, Miki Y and Tanaka H: Aurora kinase B is a predictive factor for the aggressive recurrence of hepatocellular carcinoma after curative hepatectomy. Br J Surg 95: 611-619, 2008

64. Wu CX, Wang XQ, Chok SH, Man K, Tsang SHY, Chan ACY, Ma KW, Xia W and Cheung TT: Blocking CDK1/PDK1/ $\beta$-Catenin signaling by CDK1 inhibitor RO3306 increased the efficacy of sorafenib treatment by targeting cancer stem cells in a preclinical model of hepatocellular carcinoma. Theranostics 8: 3737-3750, 2018.

65. Zhuang L, Yang Z and Meng Z: Upregulation of BUB1B, CCNB1, CDC7, CDC20, and MCM3 in tumor tissues predicted worse overall survival and disease-free survival in hepatocellular carcinoma patients. Biomed Res Int 2018: 7897346, 2018.

66. Jiang W, Zhang L, Guo Q, Wang H, Ma M, Sun J and Chen C: Identification of the pathogenic biomarkers for hepatocellular carcinoma based on RNA-seq analyses. Pathol Oncol Res 25: $1207-1213,2019$

67. Ganapathy E, Su F, Meriwether D, Devarajan A, Grijalva V, Gao F, Chattopadhyay A, Anantharamaiah GM, Navab M, Fogelman AM, et al: D-4F, an apoA-I mimetic peptide, inhibits proliferation and tumorigenicity of epithelial ovarian cancer cells by upregulating the antioxidant enzyme MnSOD. Int J Cancer 130: 1071-1081, 2012.
68. Cedó L, García-León A, Baila-Rueda L, Santos D, Grijalva V, Martínez-Cignoni MR, Carbó JM, Metso J, López-Vilaró L, Zorzano A, et al: ApoA-I mimetic administration, but not increased apoA-I-containing HDL, inhibits tumour growth in a mouse model of inherited breast cancer. Sci Rep 6: 36387, 2016.

69. Gao F, Vasquez SX, Su F, Roberts S, Shah N, Grijalva V, Imaizumi S, Chattopadhyay A, Ganapathy E, Meriwether D, et al: L-5F, an apolipoprotein A-I mimetic, inhibits tumor angiogenesis by suppressing VEGF/basic FGF signaling pathways. Integr Biol (Camb) 3: 479-489, 2011.

70. Zamanian-Daryoush M, Lindner D, Tallant TC, Wang Z, Buffa J, Klipfell E, Parker Y, Hatala D, Parsons-Wingerter P, Rayman $\mathrm{P}$, et al: The cardioprotective protein apolipoprotein A1 promotes potent anti-tumorigenic effects. J Biol Chem 288: 21237-21252, 2013

71. Oh C, Park S, Lee EK and Yoo YJ: Downregulation of ubiquitin level via knockdown of polyubiquitin gene Ubb as potential cancer therapeutic intervention. Sci Rep 3: 2623, 2013.

72. Tang Y, Geng Y, Luo J, Shen W, Zhu W, Meng C, Li M, Zhou X, Zhang S and Cao J: Downregulation of ubiquitin inhibits the proliferation and radioresistance of non-small cell lung cancer cells in vitro and in vivo. Sci Rep 5: 9476, 2015.

73. Tian Y, Ding W, Wang Y, Ji T, Sun S, Mo Q, Chen P, Fang Y, Liu J, Wang B, et al: Ubiquitin B in cervical cancer: Critical for the maintenance of cancer stem-like cell characters. PLoS One 8: e84457, 2013.

74. Valdagni R, Rancati T, Ghilotti M, Cozzarini C, Vavassori V, Fellin G, Fiorino C, Girelli G, Barra S, Zaffaroni N, et al: To bleed or not to bleed. A prediction based on individual gene profiling combined with dose-volume histogram shapes in prostate cancer patients undergoing three-dimensional conformal radiation therapy. Int J Radiat Oncol Biol Phys 74: 1431-1440, 2009.

75. Daniele B, De Vivo R, Perrone F, Lastoria S, Tambaro R, Izzo F, Fiore F, Vallone $\mathrm{P}$ and Pignata S: Phase I clinical trial of liposomal daunorubicin in hepatocellular carcinoma complicating liver cirrhosis. Anticancer Res 20: 1249-1251, 2000.

76. Brandi G, Biasco G, Mirarchi MG, Golfieri R, Di Paolo A, Borghi A, Fanello S, Derenzini E, Agostini V, Giampalma E, et al: A phase I study of continuous hepatic arterial infusion of Irinotecan in patients with locally advanced hepatocellular carcinoma. Dig Liver Dis 43: 1015-1021, 2011.

77. Tak WY, Lin SM, Wang Y, Zheng J, Vecchione A, Park SY, Chen MH, Wong S, Xu R, Peng CY, et al: Phase III HEAT study adding lyso-thermosensitive liposomal doxorubicin to radiofrequency ablation in patients with unresectable hepatocellular carcinoma lesions. Clin Cancer Res 24: 73-83, 2018.

78. Lin HC, Chen YF, Hsu WH, Yang CW, Kao CH and Tsai TF: Resveratrol helps recovery from fatty liver and protects against hepatocellular carcinoma induced by hepatitis B virus $\mathrm{X}$ protein in a mouse model. Cancer Prev Res (Phila) 5: 952-962, 2012.

79. Bhattacharya S, Mondal L, Mukherjee B, Dutta L, Ehsan I, Debnath MC, Gaonkar RH, Pal MM and Majumdar S: Apigenin loaded nanoparticle delayed development of hepatocellular carcinoma in rats. Nanomedicine 14: 1905-1917, 2018.

80. Balamurugan K and Karthikeyan J: Evaluation of luteolin in the prevention of N-nitrosodiethylamine-induced Hepatocellular carcinoma using animal model system. Indian J Clin Biochem 27: $157-163,2012$.

81. Chen F, Wang H, Zhu J, Zhao R, Xue P, Zhang Q, Bud Nelson M, Qu W, Feng B and Pi J: Camptothecin suppresses NRF2-ARE activity and sensitises hepatocellular carcinoma cells to anticancer drugs. Br J Cancer 117: 1495-1506, 2017.

82. Xu D, Jin J, Yu H, Zhao Z, Ma D, Zhang C and Jiang H: Chrysin inhibited tumor glycolysis and induced apoptosis in hepatocellular carcinoma by targeting hexokinase-2. J Exp Clin Cancer Res 36: 44, 2017.

83. Xiao W, Dong W, Zhang C, Saren G, Geng P, Zhao H, Li Q, Zhu J, Li G, Zhang S and Ye M: Effects of the epigenetic drug MS-275 on the release and function of exosome-related immune molecules in hepatocellular carcinoma cells. Eur J Med Res 18: 61, 2013.

84. Zhang B, Yin X and Sui S: Resveratrol inhibited the progression of human hepatocellular carcinoma by inducing autophagy via regulating $\mathrm{p} 53$ and the phosphoinositide 3 kinase/protein kinase B pathway. Oncol Rep 40: 2758-2765, 2018.

85. Park S, Lim J, Kim JR and Cho S: Inhibitory effects of resveratrol on hepatitis B virus X protein-induced hepatocellular carcinoma. J Vet Sci 18: 419-429, 2017.

86. Liao PC, Ng LT, Lin LT, Richardson CD, Wang GH and Lin CC Resveratrol arrests cell cycle and induces apoptosis in human hepatocellular carcinoma Huh-7 cells. J Med Food 13: 1415-1423, 2010. 
87. Notas G, Nifli AP, Kampa M, Vercauteren J, Kouroumalis E and Castanas E: Resveratrol exerts its antiproliferative effect on HepG2 hepatocellular carcinoma cells, by inducing cell cycle arrest, and NOS activation. Biochim Biophys Acta 1760 1657-1666, 2006.

88. Bishayee A, Politis T and Darvesh AS: Resveratrol in the chemoprevention and treatment of hepatocellular carcinoma. Cancer Treat Rev 36: 43-53, 2010.

89. Li Y, Cheng X, Chen C, Huijuan W, Zhao H, Liu W, Xiang Z and Wang Q: Apigenin, a flavonoid constituent derived from $P$. villosa, inhibits hepatocellular carcinoma cell growth by CyclinD1/CDK4 regulation via p38 MAPK-p21 signaling. Pathol Res Pract 216: 152701, 2020.

90. Cao Z, Zhang H, Cai X, Fang W, Chai D, Wen Y, Chen H, Chu F and Zhang Y: Luteolin promotes cell apoptosis by inducing autophagy in hepatocellular carcinoma. Cell Physiol Biochem 43: 1803-1812, 2017
91. Fan YP, Liao JZ, Lu YQ, Tian DA, Ye F, Zhao PX, Xiang GY, Tang WX and He XX: MiR-375 and doxorubicin Co-delivered by liposomes for combination therapy of hepatocellular carcinoma. Mol Ther Nucleic Acids 7: 181-189, 2017.

92. Ang C, O'Reilly EM, Carvajal RD, Capanu M, Gonen M, Doyle L, Ghossein R, Schwartz L, Jacobs G, Ma J, et al: A nonrandomized, phase II study of sequential irinotecan and flavopiridol in patients with advanced hepatocellular carcinoma. Gastrointest Cancer Res 5: 185-189, 2012.

93. Liu L, Chen X, Xie S, Zhang C, Qiu Z and Zhu F: Variant 1 of KIAA0101, overexpressed in hepatocellular carcinoma, prevents doxorubicin-induced apoptosis by inhibiting p53 activation. Hepatology 56: 1760-1769, 2012.

(c) (7) (3) This work is licensed under a Creative Commons (c) Attribution 4.0 International (CC BY-NC 4.0) License 